\title{
Acute thumb and finger instability
}

\begin{abstract}
This chapter describes the intricate bony and ligamentous anatomy of the 19 finger and thumb joints in the hand and their disruptions in all four directions. The epidemiology of the injuries, the mechanisms of the disruptions and the best way to diagnose and treat them in order to avoid the complications are explained with diagrams and clinical pearls to help the novice as well as the learned surgeon.
\end{abstract}

Keyword: Capsular injuries; CMC dislocations; Collateral ligament tear; Digital dislocations; DIP dislocations; Hand dislocations; Instability; Ligamentous injuries; Mallet finger; MCP dislocations; PIP dislocations; Sagittal band disruptions; Sports hand injuries; Stener lesion; Stiffness (digital); UCL tear; Volar plate injury 